\title{
Performance of Different Atrial Conduction Velocity Estimation Algorithms Improves with Knowledge about the Depolarization Pattern
}

https://doi.org/10.1515/cdbme-2019-0026

\begin{abstract}
Quantifying the atrial conduction velocity (CV) reveals important information for targeting critical arrhythmia sites that initiate and sustain abnormal electrical pathways, e.g. during atrial flutter. The knowledge about the local CV distribution on the atrial surface thus enhances clinical catheter ablation procedures by localizing pathological propagation paths to be eliminated during the intervention.
\end{abstract}

Several algorithms have been proposed for estimating the CV. All of them are solely based on the local activation times calculated from electroanatomical mapping data. They deliver false values for the $\mathrm{CV}$ if applied to regions near scars or wave collisions. We propose an extension to all approaches by including a distinct preprocessing step. Thereby, we first identify scars and wave front collisions and provide this information for the $\mathrm{CV}$ estimation algorithm. In addition, we provide reliable $\mathrm{CV}$ values even in the presence of noise. We compared the performance of the Triangulation, the Polynomial Fit and the Radial Basis Functions approach with and without the inclusion of the aforementioned preprocessing step. The evaluation was based on different activation patterns simulated on a $2 \mathrm{D}$ synthetic triangular mesh with different levels of noise added.

The results of this study demonstrate that the accuracy of the estimated CV does improve when knowledge about the depolarization pattern is included. Over all investigated test cases, the reduction of the mean velocity error quantified to at least $25 \mathrm{~mm} / \mathrm{s}$ for the Radial Basis Functions, $14 \mathrm{~mm} / \mathrm{s}$ for the Polynomial Fit and $14 \mathrm{~mm} / \mathrm{s}$ for the Triangulation approach compared to their respective implementations without the preprocessing step.

Given the present results, this novel approach can contribute to a more accurate and reliable CV estimation in a clinical setting and thus improve the success of radio-frequency ablation to treat cardiac arrhythmias.

Keywords: atrial conduction velocity, local activation time processing, atrial flutter

\footnotetext{
*Corresponding author: Claudia Nagel, Karlsruhe Institute of Technology, Institute of Biomedical Engineering, Karlsruhe, Germany, e-mail: publications@ibt.kit.edu

Nicolas Pilia, Laura Unger, Olaf Dössel, Karlsruhe Institute of Technology, Institute of Biomedical Engineering, Karlsruhe, Germany
}

\section{Introduction}

Atrial flutter is one of the most common cardiac arrhythmias in e.g. European countries and the USA. A standard procedure to treat this type of supraventricular tachycardia is radiofrequency ablation. Thereby, small lesions are induced on the cardiac tissue to eliminate the abnormal excitation pathways.

The localization of these pathological paths such as slow conducting tissue areas can be carried out by quantifying the cardiac conduction velocity and its direction. For this purpose, several CV estimation algorithms have already been proposed. However, all of these algorithms reported as suitable for a clinical environment are based on the local activation times (LAT) that are extracted from measured intracardiac electrograms [1]. They do neither consider that scar tissue stops the excitation propagation nor do they deliver reliable estimation values in vicinity to wave front collisions.

We propose an extension applicable to three existing algorithms by including a distinct prepocessing step. It consists of the identification of scar locations, wave collisions and low signal to noise ratio (SNR) areas. This a-priori knowledge about the depolarization pattern is then included in the CV estimation routine. The performance evaluation was carried out with three algorithms reported as suitable for the clinical use case [3-5] on a 2D triangle mesh.

\section{Methods}

\subsection{Synthetic Test Cases}

Synthetic test cases with a known ground truth CV were simulated with the Fast Marching method [6]. In this way, the estimated CV output from the algorithms could be validated with the predefined ground truth $\mathrm{CV}$ of the simulation setup.

Different activation patterns were simulated on a $2 \mathrm{D}$ equilateral triangle mesh. The mesh contained 15229 faces which composed 30096 triangular faces with a side length of $1.3 \mathrm{~mm}$. These values are chosen according to an exemplary clinical atrial mesh to create settings comparable to the actual clinical use case. 
The simulated LAT maps covered all major activation patterns that occur in practice, including the following propagation characteristics:

- a radial as well as a planar wave with a homogeneous CV set to $200 \mathrm{~mm} / \mathrm{s}$ all over the mesh (cf. Fig. 1a, 1d)

- a planar wave passing through four horizontal areas with different CVs starting with $200 \mathrm{~mm} / \mathrm{s}$ at the bottom edge and increasing in steps of $\delta_{v}=20 \mathrm{~mm} / \mathrm{s}$ (cf. Fig. 1b) and $\delta_{v}=200 \mathrm{~mm} / \mathrm{s}$ (cf. Fig. 1c)

- two focal sources at the lower left and the upper right corner of the mesh causing a wave collision on the main diagonal (cf. Fig. 1e)

- a linear horizontal scar a with a planar wave propagation in orthogonal and parallel direction with respect to the scar (cf. Fig. 1h, 1g) and with a radial wave propagation from a focal source in the lower left corner (cf. Fig. 1f)

- a box shaped scar with a gap causing delayed depolarization of the enclosed tissue (cf. Fig. 1i)

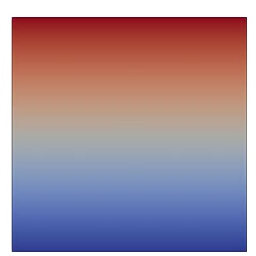

(a) Planar Wave

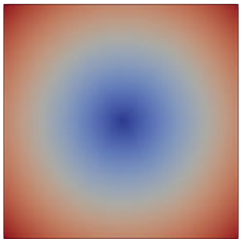

(d) Radial wave

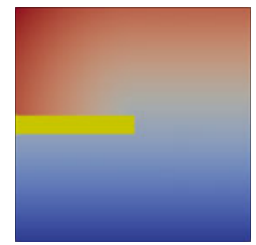

(g) Horizontal scar with parallel planar wave

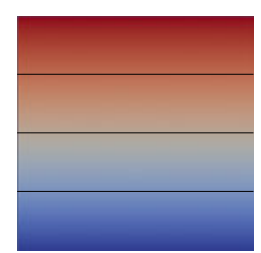

(b) $\delta_{v}=20 \mathrm{~mm} / \mathrm{s}$

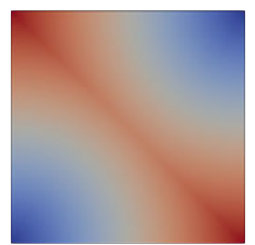

(e) Wave front collisions

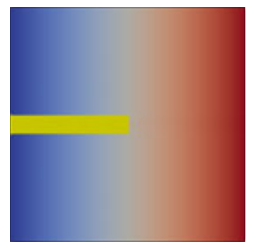

(h) Horizontal scar with vertical planar wave

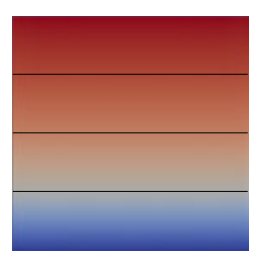

(c) $\delta_{v}=200 \mathrm{~mm} / \mathrm{s}$

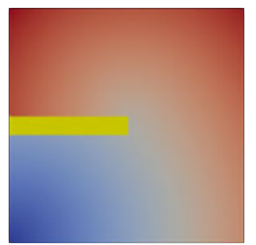

(f) Horizontal scar with spherical wave

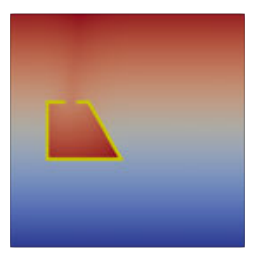

(i) Box shaped scar
Fig. 1: Synthetic 2D test cases representing the excitation patterns to be identified in a clinical environment. The ground truth CV for the Fast Marching simulation is set to $200 \mathrm{~mm} / \mathrm{s}$ unless otherwise stated. Scar nodes are marked in yellow. Blue colored areas represent early excited areas, whereas red colored areas indicate that the excitation arrives later in time.
Following the Fast Marching simulation, white Gaussian noise with an SNR of $15 \mathrm{~dB}$ and $30 \mathrm{~dB}$ was added to the spatial coordinates and the temporal LAT values. In this way the circumstances in a clinical environment, consisting of LAT measurement noise and electrode localization errors, were simulated.

Fig. 2 sketches the workflow of the adapted CV estimation routine with the inclusion of the knowledge about the pattern of depolarization. The individual processing steps are explained in more detail in the subsequent sections.

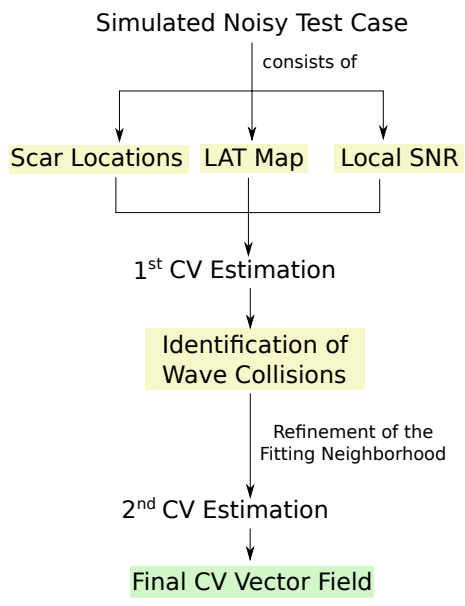

Fig. 2: Workflow for estimating the CV with noisy LAT maps by including information about scars, wave front collisions and the local SNR distribution.

\subsection{LAT Map Preprocessing}

To ensure that excitation propagation was not modeled through scar nodes, the LAT Maps were first preprocessed before applying the $\mathrm{CV}$ estimation algorithm. The knowledge about the scar locations were given by the simulation settings for the Fast Marching algorithm. Mesh edges that connected those scar nodes to any other node in the mesh were deleted. In this way, the mesh connectivities represented only valid excitation pathways and scar locations were not part of the mesh anymore.

\subsection{Robust CV Estimation with Noisy LAT Maps in the Presence of Scars}

The CV estimation was carried out with the triangulation approach and by means of surface fitting with a polynomial of order two as well as with Gaussian radial basis functions (RBF). These three approaches are well documented in literature and showed promising results in various simulation and clinical 
studies [3-5]. Some adjustments were made to ensure proper behavior around scars, wave collisions and noisy LAT areas.

This included on the one hand the choice of the local fitting neighborhood for the surface fitting approaches. The fitting neighborhood was determined according to the graph connections instead of the Euclidean distance to the surrounding points. In this way, scar nodes were excluded from the fit which would otherwise have distorted the fitted surface and yielded an inaccurate CV estimation. Furthermore, the neighborhood size was increased from 20 to 200 points if the SNR in a specific area felt below a threshold of $50 \mathrm{~dB}$. In this way, the radius of the fitting neighborhood was approximately increased by factor three. Thereby, the algorithms were provided a larger fitting region to average over noisy LAT points.

To reduce the influence of noisy LATs at the mesh nodes for the triangulation approach, the triangles to fit in a planar wave were chosen three times larger than the ones in the original triangulation mesh. Nevertheless, this procedure does not result in a decreased mesh density. Instead, the extended triangles were shifted and rotated along the original mesh and overlapping regions were assigned the average of the calculated CVs. Scar nodes were not included in the calculation process for this approach either by inhibiting the planar wave fit in an enlarged triangle that overlaps with scar regions.

\subsection{Identification of Wave Front Collisions}

The identification of wave front collisions within the activation patterns in connection with the $\mathrm{CV}$ estimation is crucial because a wave's velocity is physically not defined at a node where two wave fronts collide with each other. Therefore, it was necessary to localize these points and deliver a warning that a CV estimation at these spots is not reliable.

The wave front collisions were identified by means of the divergence operator [2]:

$$
\nabla C V=\frac{\partial C V_{x}}{\partial x}+\frac{\partial C V_{y}}{\partial y}
$$

The divergence operator provided a metric for sinks and sources within a vector field. It characterizes sources with a positive, sinks or collisions with a negative and a parallel propagation with a value around zero. The $\mathrm{CV}$ values calculated with one of the algorithms presented in the previous section were first normalized before put into equation 1 . In this way, it was guaranteed that only the direction of the $\mathrm{CV}$ vectors have an impact on the divergence and not their magnitude [1].

Hereafter, the choice of the neighborhood for the surface fitting as well as of the triangles for the triangulation approach was refined with the knowledge about collision locations. In this way, the CV estimation in a second iteration could be performed in regions where a mostly homogeneous excitation propagation prevails. In such areas the planar wave assumption holding for the triangulation approach can approximately be met. Furthermore, the fitting of a planar LAT surface with the RBF and the Polynomial Fit method can be executed more robustly compared to one of an undulating surface. Therefore, the $\mathrm{CV}$ calculation was carried out a second time taking the divergence values at each mesh point into consideration.

\section{Results}

In this study, the CV calculation for each test case was carried out for each of the three presented algorithms once with and once without the knowledge about collision and scar locations as well as with the local SNR. The mean velocity error of each experiment was calculated by averaging the absolute value of the differences between the ground truth $\mathrm{CV}$ that was set for the simulation and the estimated $\mathrm{CV}$ values for each mesh node or triangle $i$ :

$$
\operatorname{mean}(\Delta v)=\frac{1}{N} \sum_{i=1}^{N}\left|C V_{e s t, i}-C V_{g t, i}\right|
$$

The change of the mean velocity error when including the preprocessing step was quantified in the following way:

$$
\Delta e=\operatorname{mean}\left(\Delta v_{w}\right)-\operatorname{mean}\left(\Delta v_{w o}\right)
$$

The terms mean $\left(\Delta v_{w}\right)$ and mean $\left(\Delta v_{w o}\right)$ refer to the mean velocity error calculated according to equation 2 with $\left(\Delta v_{w}\right)$ and without $\left(\Delta v_{w o}\right)$ the preprocessing step, respectively. A negative value of $\Delta e$ thus implied an improvement and a positive one a deterioration of the $\mathrm{CV}$ estimation performance when including the a-priori knowledge about scars, wave collisions and the local SNR.

Tab. 1 shows that the change of the mean velocity error was negative for all test cases, both noise levels and all CV estimation algorithms.

When including a-priori knowledge, the absolute velocity errors mean $\left(\Delta v_{w}\right)$ averaged over all test cases quantified to $56 \mathrm{~mm} / \mathrm{s}(145 \mathrm{~mm} / \mathrm{s}), 24 \mathrm{~mm} / \mathrm{s}(102 \mathrm{~mm} / \mathrm{s})$ and $9 \mathrm{~mm} / \mathrm{s}(39 \mathrm{~mm} / \mathrm{s})$ for the Triangulation, the Polynomial Fit and the RBF approach in the $30 \mathrm{~dB}(15 \mathrm{~dB})$ cases.

\section{Discussion}

In each investigated case, the inclusion of the knowledge about the depolarization pattern reduced the mean velocity error in 
Tab. 1: Assessement of the mean velocity error change $\Delta e$ in $\mathrm{mm} / \mathrm{s}$ for all test cases with an SNR of $15 \mathrm{~dB}$ and $30 \mathrm{~dB}$ when including the preprocessing step. Negative values imply an improvement by including a-priori knowledge.

\begin{tabular}{lcccccc}
\hline Test Case & \multicolumn{2}{c}{ Triangulation } & \multicolumn{2}{c}{ Polynomial Fit } & \multicolumn{2}{c}{ RBF } \\
& 15dB & 30dB & 15dB & 30dB & 15dB & 30dB \\
\hline (a) Planar Wave & -35 & -56 & -25 & -25 & -83 & -34 \\
(b) $\delta v=20 \mathrm{~mm} / \mathrm{s}$ & -31 & -62 & -56 & -28 & -103 & -38 \\
(c) $\delta v=200 \mathrm{~mm} / \mathrm{s}$ & -75 & -88 & -124 & -19 & -167 & -88 \\
(d) Spherical Wave & -35 & -54 & -29 & -21 & -82 & -25 \\
(e) Wave Collisions & -29 & -61 & -14 & -39 & -87 & -41 \\
(f) Scar, Radial W. & -14 & -55 & -14 & -46 & -86 & -63 \\
(g) Scar, Parallel W. & -24 & -57 & -16 & -41 & -91 & -54 \\
(h) Scar, Vertical W. & -26 & -56 & -27 & -34 & -85 & -46 \\
(i) Box Shaped Scar & -32 & -59 & -27 & -31 & -88 & -40 \\
\hline
\end{tabular}

the mesh independent on the choice of the CV estimation algorithm (cf. Tab. 1). This implies that the inclusion of the preprocessing step definitely leads to a more robust and reliable $\mathrm{CV}$ estimation for simulated clinically relevant excitation patterns. The inclusion of a-priori knowledge has the highest impact on the $\mathrm{CV}$ estimation with the RBF approach and the lowest one for the Polynomial Fit method.

The knowledge about the scar locations are given by the simulation setup in this study. Nevertheless, they are also extractable from clinical cata. The same holds for the local signal to noise ratio. Therefore, the proposed preprocessing routine is not only suited for simulated environments but it can also improve the results of the $\mathrm{CV}$ estimation in a real clinical setting.

The identification of wave front collisions required an initial calculation of the $\mathrm{CV}$ vector field before the neighborhood selection could be refined for the second iteration of the $\mathrm{CV}$ estimation. This is a valid approach if the initial calculation of the velocity directions already delivered values of sufficient accuracy for the application of the divergence operator when scar locations and the local SNR are available. For all experiments with the lowest SNR, the median angle between the ground truth $\mathrm{CV}$ direction and the calculated $\mathrm{CV}$ direction after the first iteration amounts to $55^{\circ}, 12^{\circ}$ and $4^{\circ}$ for the Triangulation, the Polynomial Fit and the RBF approach, respectively. This estimation is of adequate precision for the Polynomial Fit and the RBF method, which is why the initial vector field estimation could be used for the application of the divergence operator before the CV speed could be assessed more precisely.

However, the use of collision detection to position the enlarged triangles for the Triangulation approach might be debatable due to the relatively high direction error after the first CV estimation iteration. The latter reflects also in the values for $\Delta e$ shown in Tab.1. The improvement for the Triangula- tion approach with an SNR of $15 \mathrm{~dB}$ is for all cases inferior compared to those of the $30 \mathrm{~dB}$ cases.

\section{Conclusion}

We showed that an additional processing step yields a more robust $\mathrm{CV}$ estimation in combination with the three investigated $\mathrm{CV}$ algorithms compared to their respective implementations as documented in literature. This fosters confidence that the knowledge about scar locations, the SNR distribution and wave collisions contributes to a sufficiently reliable $\mathrm{CV}$ calculation. In doing so, a quantitative metric can be deduced during a clinical electrophysiological study to target ablation locations for the treatment of cardiac arrhythmias.

\section{Author Statement}

Research funding: The author state no funding involved. Conflict of interest: Authors state no conflict of interest.

\section{References}

[1] C. Cantwell, C. Roney, F. Ng, et al., "Techniques for automated local activation time annotation and conduction velocity estimation in cardiac mapping," Computers in Biology and Medicine, vol. 65, Oct. 2015, pp. 229-242.

[2] T. Fitzgerald, D. Brooks, and J. Triedman, "Identification of Cardiac Rhythm Features by Mathematical Analysis of Vector Fields," IEEE Transactions on Biomedical Engineering, vol. 52, no. 1, Jan. 2005, pp. 19-29.

[3] M. Mase and F. Ravelli, "Automatic reconstruction of activation and velocity maps from electro- anatomic data by radial basis functions," in 2010 Annual International Conference of the IEEE Engineering in Medicine and Biology. Buenos Aires: IEEE, Aug. 2010, pp. 2608-2611.

[4] A. Barnette, P. Bayly, Shu Zhang, et al., "Estimation of 3-D conduction velocity vector fields from cardiac mapping data," IEEE Transactions on Biomedical Engineering, vol. 47, no. 8, Aug. 2000, pp. 1027-1035.

[5] C.D.Cantwell, C.H.Roney, R.L.Ali, et al., "A software platform for the comparative analysis of electroanatomic and imaging data including conduction velocity mapping," in 2014 36th Annual International Conference of the IEEE Engineering in Medicine and Biology Society. Chicago, IL: IEEE, Aug. 2014, pp. 1591-1594.

[6] J.A.Sethian, "A fast marching level set method for monotonically advancing fronts. "Proceedings of the National Academy of Sciences, vol. 93, no. 4, Feb. 1996, pp. 1591-1595. 